\title{
Atmospheric Electron X-ray Spectrometer Development ${ }^{1}$
}

\author{
Jet Propulsion Laboratory \\ 4800 Oak Grove Dr. \\ Pasadena, CA 91109 \\ 818-354-4321 \\ Jason.Feldman@ipl.nasa.gov \\ Jaroslava.Z.Wilcox@ojpl.nasa.gov \\ Thomas.George@ipl.nasa.gov \\ Nathan.Bridges@jpl.nasa.gov \\ David N. Barsic, Axel Scherer \\ California Institute of Technology \\ 1200 E. California Blvd. \\ Pasadena, CA 91125 \\ dnbarsic acaltech.edu \\ etcher@altech.edu
}

Jason E. Feldman, Jaroslava Z. Wilcox, Thomas George, Nathan Bridges

\begin{abstract}
The development of a portable surface elemental analysis tool based on the excitation of characteristic x-rays at ambient pressure with an electron beam is described. This instrument relies on the use of a thin electron transmissive membrane to isolate the vacuum of the electron source from the ambient atmosphere. The major advantages offered by this instrument include rapid spectrum acquisition, non-destructive evaluation of elemental composition, and high spatial resolution in comparison to similar portable instruments. The instrument proof-ofprinciple has been demonstrated by obtaining energy dispersive x-ray spectra from metal and mineral samples. Scanning Electron Microscope (SEM) experiments have been carried out to determine beam spot size and quantitative analysis limits. Modeling has been performed to study performance limits and to understand the influence of membrane and atmosphere interactions on the focused electron beam.
\end{abstract}

\section{TABLE OF CONTENTS}

1. INTRODUCTION

2. INSTRUMENT CHARACTERISTICS

3. PROOF OF CONCEPT

4. MODELING

5. CONCLUSIONS

6. REFERENCES

\section{INTRODUCTION}

The Atmospheric Electron X-ray Spectrometer $\left(\mathrm{AEXS}^{1,2}\right)$ is a novel miniature in situ instrument enabling rapid elemental analysis of samples on planetary surfaces by $x$-ray fluorescence (XRF). Three XRF excitation sources are currently used to analyze surface composition in ambient pressure: (i) radioactive alpha particle sources ${ }^{3}$, (ii) x-ray sources, or (iii) a focused ion beam. Here, we present the use of a fourth technique, the excitation of characteristic XRF using a focused electron beam, which we have demonstrated in ambient atmosphere. Electron beam excited Energy Dispersive X-ray Analysis (EDX) is a widely accepted technique for determining the elemental surface composition of samples in vacuum, for example analytical SEM. EDX has not been previously demonstrated in ambient atmosphere due to the difficulty of generating and transmitting electron beams through the atmosphere. The AEXS instrument takes its origins from a novel concept of a "Miniature Electron Microscope without Vacuum Pumps" proposed by T. George. ${ }^{3}$ This concept described the means towards obtaining electron beam-excited XRF in air from samples by using miniature electron sources encapsulated by electron-transmissive membranes. In this work we present the results of our work towards the development of the AEXS based on this principle, and shown schematically in Figure 1.

The instrument consists of an electron column encapsulated by an electron transmissive membrane, an EDX detection system, a high voltage power supply, and an analyzer. The electron source currently used is a miniature electron gun manufactured by Lexel Imaging Systems, Incorporated, which includes a thermionic emitter and electrostatic optics. The emitter generates the electron beam, which is subsequently accelerated to an energy of 10 to $20 \mathrm{keV}$ and focused by the electrostatic optics onto the membrane. The electron beam transmitted through the membrane impinges on a sample located outside the membrane, in the planetary

\footnotetext{
${ }^{1} 0-7803-6599-2 / 01 / \$ 10.00 \bigcirc 2001 \mathrm{IEEE}$
} 


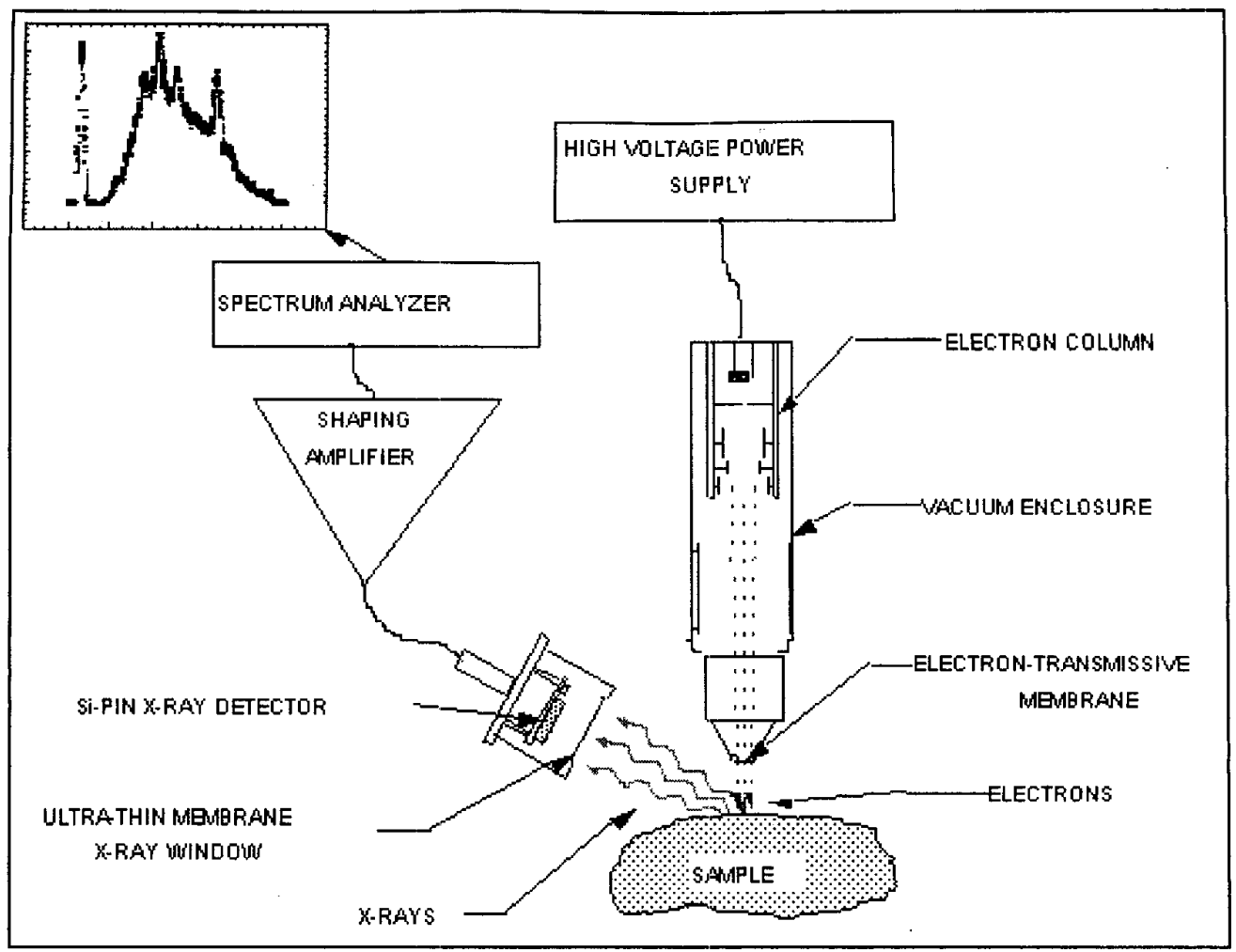

Figure 1. The AEXS instrument concept with the major components: encapsulated electron source with microfabricated electron-transmissive membrane, power supply, and x-ray detector system, and analyzer.

atmosphere. Characteristic $x$-rays are generated from the irradiated portion of the sample.

The $\mathrm{x}$-ray detector system is a silicon $p$ type-intrinsic- $n$ type (PIN) photodiode based system manufactured by Amptek Incorporated and used on the Mars Pathfinder Alpha Proton $\mathrm{X}$-ray Spectrometer APXS ${ }^{4}$ instrument. The electrons also ionize the planetary atmosphere, ensuring a return current path. ${ }^{5}$ The elemental composition of the irradiated spot is determined by analyzing the electron-beam excited XRF using the EDX analyzer system.

\section{INSTRUMENT CHARACTERISTICS}

The transmission of an electron beam in ambient atmosphere enables a new approach for in situ XRF measurements of planetary surfaces. In the past, XRF instruments have proved to be invaluable for determining the elemental makeup of the surface of a planetary body. To date, all in situ missions, including Surveyor, Viking 1, Viking 2, Mars Pathfinder, and the Soviet Venera/Vega missions have carried some form of XRF instrument. XRF instruments are also being considered for future Mars missions. Previous planetary XRF instruments have used radioactive sources to excite x-rays from planetary materials. A comparison of the AEXS, an X-ray excitation based XRF instrument, and the
Alpha Proton X-ray Spectrometer (APXS) is shown in Table 1.

Small irradiated spot size. An instrument providing a 100 $\mu \mathrm{m}$ to several $\mathrm{mm}$ spot size on the irradiated sample should be able to determine local elemental composition of rocks and soil, which has not yet been achieved on another planet. For comparison, the APXS measured a spot approximately $4 \mathrm{~cm}$ in diameter. The small spot size will be particularly effective when used in conjunction with other instruments, such as high resolution imaging systems boresighted (examining the same region of the sample) with the electron beam.

Rapid spectrum acquisition. The estimated x-ray fluorescence spectrum acquisition time for the AEXS with a $10 \mu \mathrm{A}$ electron beam is under a minute as compared to 10 hours required by the APXS. Such short spectrum acquisition times (resulting in low energy consumption per spectrum) will enable multiple readings of a rock or outcrop, thereby assessing bulk mineralogy and heterogeneity.

Alternatively, this instrument can be used to rapidly scan several samples, effectively providing a quick look, petrologic field reconnaissance of a region. 


\begin{tabular}{|c|c|c|c|}
\hline Property & AEXS & XRF/XRD (proposed) & APXS (flight) \\
\hline Excitation particles & Electrons & $\mathrm{x}$-ray photons & $\alpha$-particles \\
\hline Particle flux & $6 \times 10^{13} / \mathrm{s} \quad(10 \mu \mathrm{A})$ & $\begin{array}{l}\text { Primary current: } 0.3 \mathrm{~mA} ; \\
\text { X-ray photons: } 2 \times 10^{12} / \mathrm{s}\end{array}$ & $2 \times 10^{9} / \mathrm{s}(50 \mathrm{mCi})$ \\
\hline Power & $5 \mathrm{~W}$ (peak operation) & $13 \mathrm{~W}$ & $0.34 \mathrm{~W}$ \\
\hline $\begin{array}{l}\text { Excitation efficiency: (\# } \\
\text { X-ray photons per } \\
\text { excitation particle) }\end{array}$ & $5 \times 10^{-6}$ & $1.4 \times 10^{-5}$ & $5 \times 10^{-6}$ \\
\hline $\begin{array}{l}\text { Geometrical } \\
\text { efficiency }^{(1)}\end{array}$ & $>10^{-4}$ & $10^{-6}$ to $10^{-5}$ & $10^{-4}$ \\
\hline \#X-ray photons/second & $>2 \times 10^{4}$ & $10^{2}$ to $10^{3}$ & $\sim 1$ \\
\hline $\begin{array}{ll}\text { Spectrum } & \text { acquisition } \\
\text { time }^{(2)} & \\
\end{array}$ & 10 seconds & 5 minutes & 10 hours \\
\hline Energy per acquisition & $50 \mathrm{~J}$ & $5,000 \mathrm{~J}$ & $10,000 \mathrm{~J}$ \\
\hline Irradiated spot size & $\begin{array}{l}\text { Controlled by beam } \\
\text { focusing and working } \\
\text { distance, } \mathbf{m m}^{2}-\mathbf{c m}^{2} \text {. }\end{array}$ & $\begin{array}{l}\sim 4 \mathrm{~cm}^{2} \\
\text { (at } 2 \mathrm{~cm} \text { working distance) }\end{array}$ & $\sim 20 \mathrm{~cm}^{2}$ \\
\hline
\end{tabular}

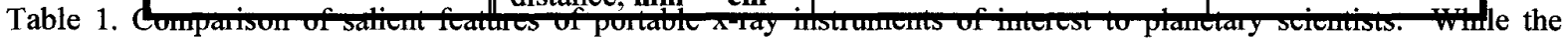
AEXS is comparable in mass (approximately $1 \mathrm{~kg}$, not shown in the table) with the other instruments, it offers two potential advantages: short spectrum acquisition time and improved spatial resolution. A consequence of the short spectrum acquisition time is that the energy expended by the instrument per acquired spectrum is also considerably less than for the other techniques.

Short penetration depth. The $\mu \mathrm{m}$-scale penetration depth of the electron beam in materials will allow surface coatings and weathering rinds on rocks to be studied with minimal mixing effects from deeper material. Measurements of unaltered surfaces will require prompt sampling of freshly cored or broken rocks, activities that are planned in several future Mars sampling missions.

Variable Spatial Resolution. The AEXS working distance (distance from the AEXS membrane to the sample) on Mars is predicted to be as much as $1 \mathrm{~cm}$. The transmitted electron beam is approximately cone shaped, so the spot size can be varied by changing the working distance. The cone angle is determined by the divergence of the electron beam due to elastic scattering effects in the membrane and the planetary atmosphere. This possibility of varying the spatial resolution is important for astrobiological investigations where large area contextual information is obtained prior to performing a higher resolution analysis.

\section{Proof of CONCEPT}

The proof of concept of the AEXS has been demonstrated by using laboratory apparatus with the same components as shown in Figure 1, incorporating the addition of a turbomolecular high vacuum pump to maintain the vacuum enclosure pressure at approximately $10^{-6}$ Torr (Figure 2.). The electronics include the Amptek ${ }^{6} \mathrm{x}$-ray detector cooled to

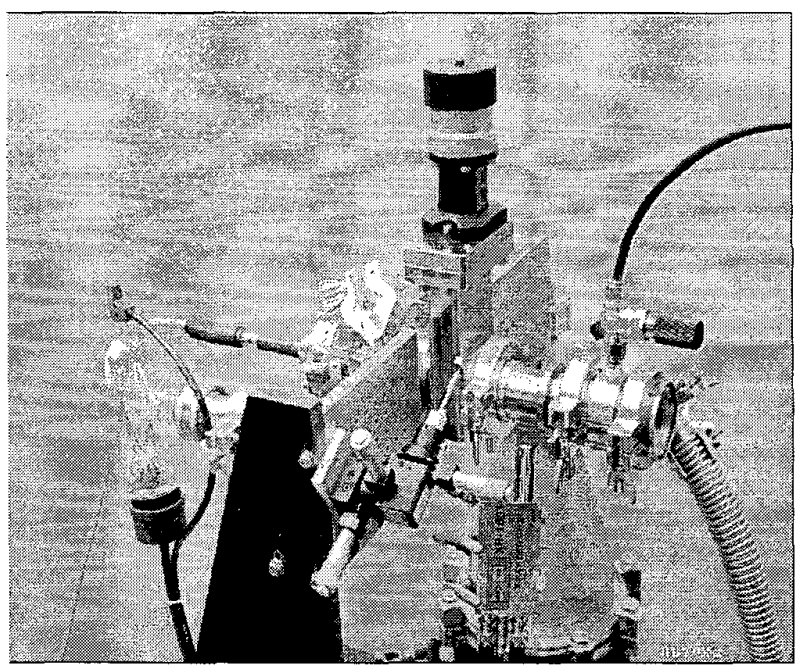

$255 \mathrm{~K}$, a pulse shaping amplifier, a high voltage power Figure 2. AEXS laboratory apparatus mounted on turbomolecular pump (not shown).

supply to accelerate and focus the electrons, and an analyzer to acquire the spectrum. The electron-transmissive encapsulation membrane is shown in figure 3 . The membrane consists of a $200 \mathrm{~nm}$ thick film of Silicon Nitride (SiN) grown using low pressure chemical vapor deposition under non-stoichiometric, silane rich conditions to produce a low tensile stress, pinhole free film on both sides of a 400 $\mu \mathrm{m}$ thick silicon wafer. One side of the silicon wafer is coated with photoresist, and $1.5 \mathrm{~mm} \times 1.5 \mathrm{~mm}$ openings are 


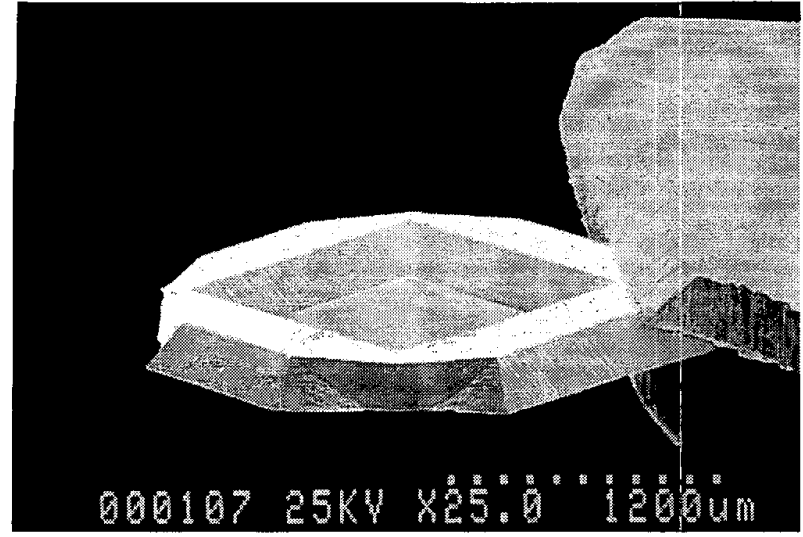

Figure 3. Scanning electron micrograph of a microfabricated Silicon Nitride membrane. Shown in the micrograph is the membrane covering the square cavity etched into a silicon frame.

created using optical photolithography. The SiN film within these openings is removed with reactive ion etching. The photoresist is then subsequently removed and $\mathrm{SiN}$ serves as a masking material for the wet chemical etching of silicon using potassium hydroxide $(\mathrm{KOH})$ solution to create a cavity bounded by the etch-resistant top SiN film on the backside. Thus the SiN "etch stop" forms the electron-transmissive encapsulation membrane and is held in place by a silicon support frame (Figure 3). The frame is then attached to a stainless steel flange using Epotech ${ }^{7}$ type H2OE silver epoxy. The final membrane "aperture" size is $1 \mathrm{~mm} \times 1 \mathrm{~mm}$.

Electron transmission through the membrane was first confirmed by introducing a sample coated with a $\mathrm{ZnO}$ phosphor into the path of the electron beam in air. XRF measurements were conducted simultaneously using the

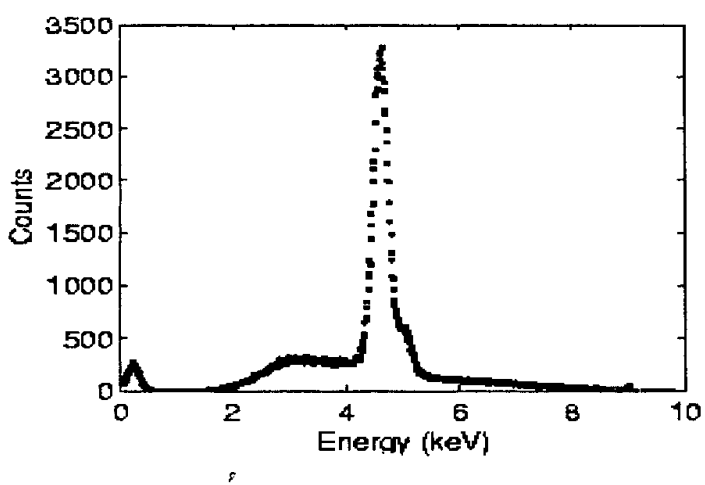

solid state $x$-ray detector. To verify that the observed fluorescence was indeed excited by the electrons, a $5 \mu \mathrm{m}$ thick polycarbonate film was inserted between the membrane and the target, resulting in a strong reduction of the characteristic $x$-ray fluorescence. When, however, the polycarbonate film was inserted between the target and the $\mathrm{X}$-ray detector, only a very slight reduction was observed. The polycarbonate film when placed in the path of the electron beam reduces the transmitted electron fraction significantly, thereby reducing the corresponding $\mathrm{x}$-ray fluorescence. When placed instead between the sample and the x-ray detector the x-ray fluorescence suffers much less attenuation. These results confirm that the excitation particles are electrons.

The irradiated spot size was roughly determined by observing the luminous spot on the phosphor screen. A diameter of between $0.5 \mathrm{~mm}$ and $1.5 \mathrm{~mm}$ was observed at 2 $\mathrm{mm}$ working distance. It should be noted that both electrons and $x$-rays can excite luminescence in the phosphor. Due to the inevitable presence of spurious $x$-rays arising from electron beam excitation prior to irradiating the phosphor, the measured diameter provides us with an upper limit to the electron spot size.

The membrane properties were investigated in detail due to its critical role in the AEXS. A series of experiments determined that the $1 \mathrm{~mm} \times 1 \mathrm{~mm}$ membrane is capable of isolating high vacuum, is able to withstand differential pressure in excess of one atmosphere, survive vibrational shocks of a magnitude to be expected during a planetary mission, and have high electron transmission.

Next, x-ray fluorescence experiments were carried out by

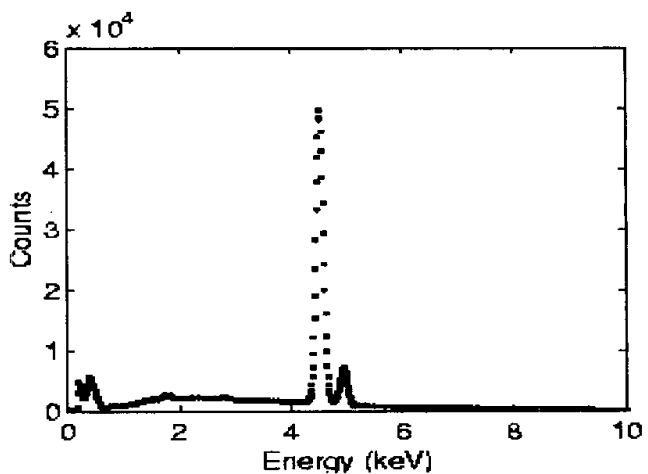

(b)

Figure 4. X-ray fluouescence spectra obtained from a pure Ti target (a) in air by the AEXS test setup and (b) in a SEM operated at $10 \mathrm{kV}$ respectively. The two Ti peaks seen in the SEM-acquired spectrum at about $4 \mathrm{kV}$ are not completely resolved by the AEXS due to the poorer resolution $(\sim 250 \mathrm{eV})$ of the X-ray detector. The background counts are also higher in the AEXS spectrum due to the increased scattering of the electron beam, primarily by the electron-transmissive membrane. 
placing suitable targets in the path of the electrons in air. The characteristic $x$-ray fluorescence generated from the target was analyzed to determine the elemental composition of the irradiated spot.

The energy scale of the $\mathrm{x}$-ray fluorescence spectrum was calibrated using known x-ray fluorescence peaks obtained from a radioactive Americium sample. The energy values of these x-ray peaks were determined using the EDX system in an SEM. The energy-calibrated spectra were used to identify known spectral peaks in the acquired data. The calibrated system was used to analyze the AEXS generated XRF from metal and mineral targets in atmosphere. One of these targets was a $99.99 \%$ pure Titanium sample mechanically polished with 800 grit sandpaper. The sample was placed $2 \mathrm{~mm}$ from the membrane, and the $\mathrm{x}$-ray fluorescence spectrum was compared with obtained from the same target in an SEM EDX analysis system. Figures 4a and $4 \mathrm{~b}$ show this comparison. Figure $4 \mathrm{a}$ shows the spectrum taken by the AEXS, and Figure $3 \mathrm{~b}$ shows the spectrum obtained in the SEM. Note that the two clearly discernible peaks (Titanium $K \alpha$ and $K \beta$ ) in Figure $4 \mathrm{~b}$ are observed as a single, slightly shifted peak with a small shoulder in Figure 4a. The peaks are better resolved in the SEM due to the greater sensitivity of a liquid nitrogen $(77 \mathrm{~K})$ cooled $\mathrm{x}$-ray detector. The AEXS x-ray detector is cooled with Peltier cooling to $255 \mathrm{~K}$, with the best obtainable $\mathrm{x}$-ray peak width being approximately $250 \mathrm{eV}$.

\section{MODELING}

Electron transmission through the membrane and ambient atmosphere was modeled using Monte Carlo simulations ${ }^{8}$ and was experimentally validated using both AEXS and SEM experiments. Figure 5 shows the predicted electron transmission and beam divergence due to scattering events as a function of the working distance between the membrane and the target at one atmosphere pressure for a $200 \mathrm{~nm}$ thick $\mathrm{SiN}$ membrane. For example, the predicted fraction of transmitted electrons is $80 \%$ and the predicted irradiated spot diameter is $0.6 \mathrm{~mm}$ for $10 \mathrm{keV}$ electrons at a working distance of $1.0 \mathrm{~mm}$. At a $2.0 \mathrm{~mm}$ working distance the predicted diameter increases to $1 \mathrm{~mm}$. As expected, the electron transmission increases and the spot size decreases with increasing electron energy. Note that within the experimental error, the measured spot size at $2.0 \mathrm{~mm}$ agrees with theoretical predictions.

\section{CONCLUSIONS}

In summary we have demonstrated rapid acquisition of spatially resolved $\mathrm{x}$-ray spectra for surface elemental analysis by measurement with the prototype AEXS
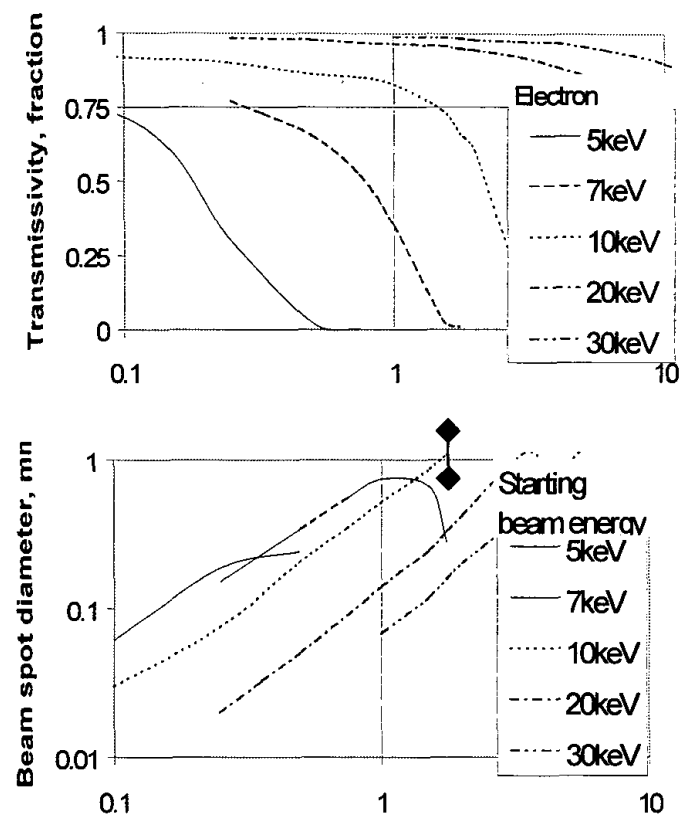

Atmosphere thickness, $\mathbf{m m}$

Figure 5. Predicted electron beam transmission and spot size as a function of the working distance at one atmosphere pressure for a 200 $\mathrm{nm}$ thick SiN membrane. Note that the measured spot size $(\bullet$ designates experimental data taken at $10 \mathrm{keV}$ and $2 \mathrm{~mm}$ working distance) agrees with the predicted spot size within the experimental error.

instrument. Using a $10 \mathrm{keV}$ electron beam and a $2 \mathrm{~mm}$ working distance in one atmosphere, the irradiated spot size was between $0.5 \mathrm{~mm}$ and $1.5 \mathrm{~mm}$, and the analysis time was as short as 100 seconds. We expect this new analysis tool to find its place among other ambient pressure surface analysis systems, such as external micro ion-beam analysis $(\mathrm{X}$ MIBA), external nuclear reaction analysis $\left(X-N R A^{9}\right)$, external Rutherford backscattering spectrometry (X-RBS), and XRF instruments. As a point of comparison, commercially available portable instruments, such as the MCA $-4000^{10}$, analyze surface regions of dimensions of several square centimeters. Comparable laboratory techniques such as X-MIBA provide focused beams for surface analysis with spatial resolutions as small as several square $\mu \mathrm{m}$ but require large stationary instruments. The potential for miniaturization and the spatial resolution of the AEXS fills the gap between non-portable, high resolution laboratory instruments and portable, low resolution in situ instruments such as the APXS used in the 1997 Mars Pathfinder mission. Our models predict that the performance of the AEXS will be improved significantly by increasing the energy of the irradiating electrons, by reducing the working distance, and by working in reduced atmospheres such as Mars ambient. In Mars atmosphere (approximately 7 Torr) a $25 \mathrm{keV}$ beam has been predicted ${ }^{11}$ 
to resolve areas as small as $100 \mu \mathrm{m}$ at $2 \mathrm{~mm}$ working distances, thus offering the potential for a compact instrument for performing rapid in-situ surface analysis at medium spatial resolution.

\section{REFERENCES}

[1] J.E. Feldman, J.Z. Wilcox, T. George, D. Barsic, and A. Scherer, "Elemental Surface Analysis at Ambient Pressure by Electron-Induced X-ray Fluorescence", accepted for publication by Rev. Sci. Instr., 2000.

[2] J.E. Feldman, T. George, and J.Z. Wilcox, "Atmospheric Electron X-ray Spectrometer" Patent application submitted

[3] T. George, "Miniature Electron Microscopes without Vacuum Pumps", NASA Tech Briefs, NPO-20335, Aug. '98.

[4] Rieder R, Wanke H, Economou T, et al., "Determination of the chemical composition of Martian soil and rocks: The alpha proton X ray spectrometer," $J$. Geophys Res-Planet 102: (E2) 4027-4044 FEB 251997

[5] G. D. Danilatos, Scanning 4, 9 (1981); Adv. Elect. \& Elect. Phys. 71, 109 (1988).

[6] Amptek Inc., 6 De Angelo Drive, Bedford, MA, USA.

[7] Epoxy Technology, 14 Fortune Dr., Billerica, MA, USA.

[8] D. Joy, "Monte Carlo Modeling for Electron microscopy and Microanalysis," Oxford University Press, 1995.

[9] B.L. Doyle, D. S. Walsh, and S. R. Lee, Nucl. Instrum. and Methods in Physics Research B 54, 244, 1991.

[10] XRF Corporation, 490 Broadway, Somerville, MA, USA.

[11] J.Z. Wilcox, "Simulation of Transmissivity of Electron Beam in Mars Atmosphere", JPL Internal Document, 1997.

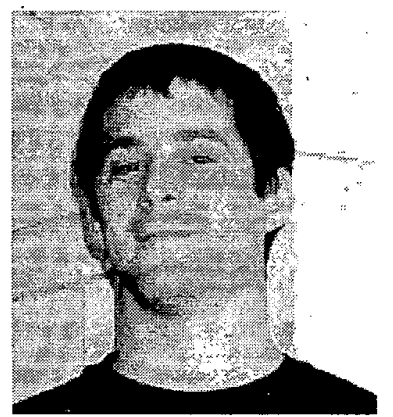

Jason Feldman is a member of the engineering staff at the Device Research and Application Section at JPL. . He received his $B A$ in Physics from the University of California at Berkeley. At Lawrence Berkeley National Laboratory, he worked with Professor Paul Richards on the Millimeter Anisotropy Experiment (MAX). In addition to his key project - the design, fabrication, and implementation of high speed radiation-hard serial data communications systems for a high altitude NASA-NSBF balloon-borne telescope, he was responsible for setting up a new laboratory and calibration facility, optimization of the analog electronics, and coordination of systems compatibility with collaborators. He joined Caltech in 1998 as an assistant engineer with Professor Axel Scherer, and JPL in 1999. He invented the
Atmospheric Electron X-ray Spectrometer with T. George and J. Z. Wilcox, and leads the experimental development of this instrument in collaboration with them. He develops new instruments and sensors for in-situ planetary exploration, bringing them from the conceptual phase to laboratory research and field operation. Jason brings a wealth of engineering expertise and practical experience in the area of design, fabrication and testing of vacuum, mechanical, electronic, and cryogenic systems. He was the cognizant engineer for the Patch Plate dust adhesion experiment, to be flown on the Mars '03 lander as part of the MECA payload. He is currently the mechanical engineer for the Cryobot Active Thermal Probe development project., which is developing a robotic probe to carry out sub-glacial exploration in Antarctica.

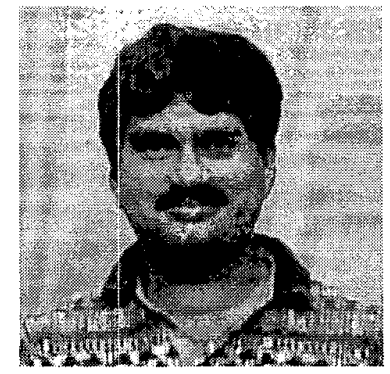

Thomas George is the Supervisor of the MEMS Technology Group at the Device Research and Application Section at JPL. He invented the concept of $a$ pump-less microfabricated electron microscope and is a co-inventor with J. Feldman and J. Z. Wilcox of the AEXS instrument. He received his $P h D$ in Materials Science from the University of California at Berkeley (UCB). Prior to joining JPL he worked on the transmission electron microscopy of semiconductor materials as a postodoctoral researcher at UCB and Intel Corporation, as well as the the Nagoya Institute of Technology, Japan. He joined JPL in 1990, where he set up and operated an HRTEM laboratory and managed tasks aimed at the development of AlGaN-based, solar-blind, ultraviolet detectors. He has successfully commercialized a JPLdeveloped, bulk Si-micromachined tunneling infrared detector. He currently manages the MEMS Technology Group with diverse MEMS-based projects and has also initiated and manages programs aimed at the development of the AEXS and a Force Detected NMR microinstrument for planetary exploration. He has authored/co-authored over 40 archival journal publications and holds 4 U.S. patents.

Jaroslava Z. Wilcox is a Member of the Technical Staff at the Device Research and Applications Section at JPL. She is a co-inventor with $T$. George and J. Feldman of a miniature ring orbitron ion pump for electron microscope applications as well as the AEXS concept. She received her $P h D$ in Solid State Physics at the University of California at Los Angeles. She taught at Tufts University for two years, and in 1974 joined TRW Space Systems where worked on inter-modulation processes in coaxes, millimeter-wave imaging, a novel phased array SAW RF spectrum analyzer, 
and electro-optics: She was the principal investigator of QWIP IR sensors program, was the program manager of the program for the development of GaAlAs solar cells, and a task leader for several diode laser development programs.

She designed and analyzed performance of $M Q W$ diode lasers and arrays of diode lasers for coherent emission and for high power (including heat removal and powering) for pumping solid state lasers. Dr. Wilcox joined JPL in 1990, where she provided support for the development of vibrational micro-gyroscope and other MEMS devices.

More recently, she was involved in the calibration of the evolved water experiment for DS2 New Millenium mission, and her current research is in the area of micro-instruments for in-situ observations. She received several technical achievement awards, was listed in "American Men and Women in Science" and "Who is Who in Technology Today", has published over 90 papers in refereed journals, has given several invited and numerous contributed talks at professional meetings, and holds several patents in the area of solid state devices.

Nathan Bridges has been a staff scientist in the Geology and Geophysics Research Element at Caltech's Jet Propulsion Laboratory since April, 2000. Prior to that he was a Caltech post-doc at JPL for three years. Bridges earned his Ph.D. in geology from the University of Massachusetts in 1997. He specializes in the study of terrestrial planet surfaces using image, multispectral, and analytical datasets. Bridges, 34,lives in Alhambra, CA, and is married with one child.

David Barsic is a graduate student in the Department of Electrical Engineering at the California Institute of Technology. He received his BS degree in Electrical Engineering from the University of Iowa in 1997. He is currently pursuing research in integrated optics and nanofabrication of MEMS devices.

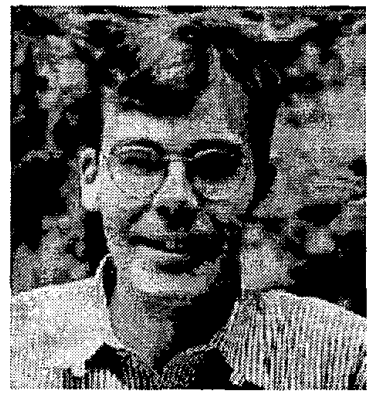

Axel Scherer is a professor of electrical engineering, applied physics and physics at Caltech. He received his $P h D$ from the New Mexico Institute of Mining and Technology in 1985 and worked at Bellcore in microfabrication of optoelectronic materials. In 1993, he joined Caltech and formed a nanofabrication research effort centered on optical, magnetic, electronic and microfluidic microstructures. Professor Scherer's research effort includes the development and application of new microfabrication methods. In the past, Professor Scherer has pioneered the development vertical cavity and photonic crystal microlasers through advanced design, crystal growth and microfabrication. Microfabrication was also used to construct new optical waveguide interferometers and spectrometers for fiber communication applications and photonic crystal waveguides for Si optical interconnects. Fundamentally new structures, such as the 2-D and 3-D photonic bandgap structure were also constructed in his laboratory and have resulted in the world's smallest laser. Professor Scherer has explored the limits of nanofabrication by defining $7 \mathrm{~nm}$ wide pillars, 10 $\mathrm{nm}$ wide lines and $35 \mathrm{~nm}$ pitch gratings with electron beam lithography. . The technology used to fabricate these nanostructures are presently used for integrated optics, dense magnetic storage and, replication molded microfluidic "laboratories" to size and sort molecules and cells. 